\title{
Preliminary evaluation of a brain PET insertable to MRI
}

\author{
Gyuseng Cho ${ }^{1 *}$, Yong Choi ${ }^{2}$, Jae Sung Lee ${ }^{3}$, Hyun Joon An ${ }^{3}$, Jin Ho Jung ${ }^{2}$, Hyun Wook Park', Chang Hyun Oh', \\ Kyeongjin Park', Kyung Taek Lim', Minsik Cho ${ }^{1}$, Woo Suk Sul ${ }^{4}$, Hyoungtaek Kim', Hyunduk Kim ${ }^{1}$ \\ From PSMR14: 3rd Conference in PET/MR and SPECT/MR \\ Kos Island, Greece. 19-21 May 2014
}

Department of Nuclear and Quantum Engineering, Korea Advanced Institute of Science and Technology, Daejeon 305-701, South Korea
There is a new trend of the medical image that diagnoses a brain disease as like Alzheimer dementia. The first qualified candidate is a PET-MRI fusion modality because MRI is a more powerful anatomic diagnosis tool than other modalities. In our study, in order to solve the high magnetic field from MRI, the development was consisted with four main items such as photo-sensor, PET scanner, MRI head-coil and attenuation correction algorithm development.

In the case of a silicon based Geiger-mode Avalanche Photo Diode (GADP), its pixel dimension is in $3 \times 3 \mathrm{~mm}^{2}$. Fabricated GAPD has a high geometric fill-factor with quenching resistors of the high resistive poly-silicon layer and a high gain $10^{6}$. PET scanner was consisted of 72 detector modules arranged in a ring of $390 \mathrm{~mm}$ diameter. Each detector module was composed of a $4 \times 4$ array GAPD coupled with an array LYSO. The signals from each PET module were fed into preamplifiers using a $3 \mathrm{~m}$ long flat cable and outputs were fed into field programmable gate array (FPGA)embedded data acquisition (DAQ) boards. A high-pass quadrature birdcage coil for high uniformity was developed in order to minimize the signal loss when it combines with PET module. In order to quickly and effectively fuse the taken image from each modality, we study a reconstruction and attenuation correction algorithm for PET images using MRI data. Finally, each component was integrated at the inside of 3 T MRI.

The preliminary test was performed while the PET-MRI system is operated simultaneously. We obtained a good performance of PET scanner that is the $16 \%$ energy resolution and the $3.0 \mathrm{~mm}$ spatial resolution. Also the PET's sensitivity in the center of field of view is a $1.2 \% \mathrm{cps} / \mathrm{Bq}$. In the case of a primary characteristic of MRI, the spatial resolution and the uniformity is a $1.0 \mathrm{~mm}(\mathrm{~T} 1, \mathrm{~T} 2)$ and $91 \%(\mathrm{~T} 1) / 87 \%(\mathrm{~T} 2)$ respectively.

The preliminary results indicate that the GAPD silicon photo sensor is excellently operated under the strong magnetic field and the developed PET-MRI system can provide high-quality PET and MRI images. Finally, an experiment of performance evaluation of a human brain of 3 candidates was conducted.

\section{SpringerOpen ${ }^{\circ}$}

(c) 2014 Cho et al; licensee Springer This is an Open Access article distributed under the terms of the Creative Commons Attribution License (http://creativecommons.org/licenses/by/4.0), which permits unrestricted use, distribution, and reproduction in any medium, provided the original work is properly cited. 


\section{Authors' details}

'Department of Nuclear and Quantum Engineering, Korea Advanced Institute of Science and Technology, Daejeon 305-701, South Korea. ${ }^{2}$ Department of Electronic Engineering Sogang University, Seoul 121-742, South Korea.

${ }^{3}$ Department of Nuclear Medicine, Seoul National University, Seoul 110-744, South Korea. ${ }^{4}$ National NanoFab Center, Deajeon 305-806, South Korea.

Published: 29 July 2014

doi:10.1186/2197-7364-1-S1-A13

Cite this article as: Cho et al.: Preliminary evaluation of a brain PET insertable to MRI. EJNMMI Physics 20141 (Suppl 1):A13.

Submit your manuscript to a SpringerOpen ${ }^{\circ}$ journal and benefit from:

- Convenient online submission

$\checkmark$ Rigorous peer review

- Immediate publication on acceptance

- Open access: articles freely available online

- High visibility within the field

- Retaining the copyright to your article

Submit your next manuscript at $\boldsymbol{s p r i n g e r o p e n . c o m ~}$ 